\title{
Successful Therapeutic Management of Concurrent Infection of Babesiosis and Theileriosis in a Holstein Friesian Calf
}

\author{
R. Ramyadevi" and K. K. Ponnuswamy \\ Department of Veterinary Clinical Medicine, Veterinary College and Research Institute, \\ Namakkal - 637 002, India \\ *Corresponding author
}

\begin{abstract}
A B S T R A C T
Keyw ords

Babesiosis,

Theileriosis,

Concurrent

infection,

Antiprotozoal

drugs, Blood

transfusion

Article Info

Accepted:

15 July 2020

Available Online:

10 August 2020

A one year old female Holstein Friesian calf was presented with a history of acute onset of anorexia, haemoglobinuria and pyrexia with severe tick infestation. Clinical examination revealed elevated rectal temperature $\left(40^{\circ} \mathrm{C}\right)$, icteric mucous membrane, enlarged prescapular and precrural lymphnodes, tachycardia and tachypnea. Haematological examination showed marked anaemia with low hemoglobin and hematocrit values. Peripheral blood smear examination revealed the presence of piroplasms of Babesia bigemia and Theileria annulata. The animal was treated with diminazene aceturate and buparvaquone along with supportive treatment of oxytetracycline, iron sorbitol and vitamin B complex injections for six days. Blood transfusion was done on third day. Animal recovered uneventfully after successful therapeutic management with antiprotozoal drugs and blood transfusion.

\section{Introduction}

Theileria and Babesia species are tick-borne haemoprotozoan parasites of vertebrates that have a severe impact on livestock production, mainly cattle and small ruminants, in tropical and subtropical areas (Mehlhorn and Schein, 1984). The diagnosis of piroplasm infections is based on clinical findings and microscopic examination of Giemsa-stained blood smears. This technique is usually adequate for detection of acute infections (Friedhoff and Bose, 1994). This article reports the

successful therapeutic management of concurrent infection of Babesiosis and Theileriosis with antiprotozoal drugs and blood transfusion in a Holstein Friesian calf.

\section{Materials and Methods}

A one year old Holstein Friesian calf was presented to the Large Animal Medicine Unit, Teaching Veterinary Clinical Complex, Veterinary College and Research Institute, Namakkal with a history of anorexia, hemoglobinuria, pyrexia with severe tick
\end{abstract}


infestation. Clinical examination revealed elevated temperature $\left(40^{\circ} \mathrm{C}\right)$, icteric mucous membrane, enlarged prescapular and precrural lymphnodes, tachycardia and tachypnea. The haematological examinations were carried out as per standard procedure. The urine sample from the cow was also subjected to routine pathological examination as per the standard technique (Benjamin, 1998).

\section{Results and Discussion}

On haematological analysis, marked anaemia was noticed with low hemoglobin $(2.6 \mathrm{~g} / \mathrm{dL})$ and hematocrit (10\%) values. Microscopic examination of Giemsa stained peripheral blood smear revealed the presence of piroplasms of Babesia bigemia and Theileria annulata (Fig. 1). The case was diagnosed as concurrent infection of Babesiosis and Theileriosis.

The animal was treated with diminazene aceturate (@ 3.5 mg/kg BW, IM), buparvaquone (@2.5 mg/kg BW, IM) and Ivermectin (@ $0.2 \mathrm{mg} / \mathrm{kg}$ BW, SC). Supportive therapy was done with oxytetracycline (@20 mg/kg BW, IV) with $0.9 \%$ sodium chloride injection, iron sorbitol injection and vitamin $\mathrm{B}$ complex injections for five days. Animal was transfused with fresh whole blood (@10-15 ml/kg BW) on third day without any anaphylactic reaction. Post transfusion hemoglobin and hematocrit values were $5.0 \mathrm{~g} / \mathrm{dL}$ and $16 \%$, respectively. The animal started to take feed and water from third day of treatment and recovered unevenfully.

Fig.1 Blood smear showing piroplasms of Babesia bigemina and Theileria annulata

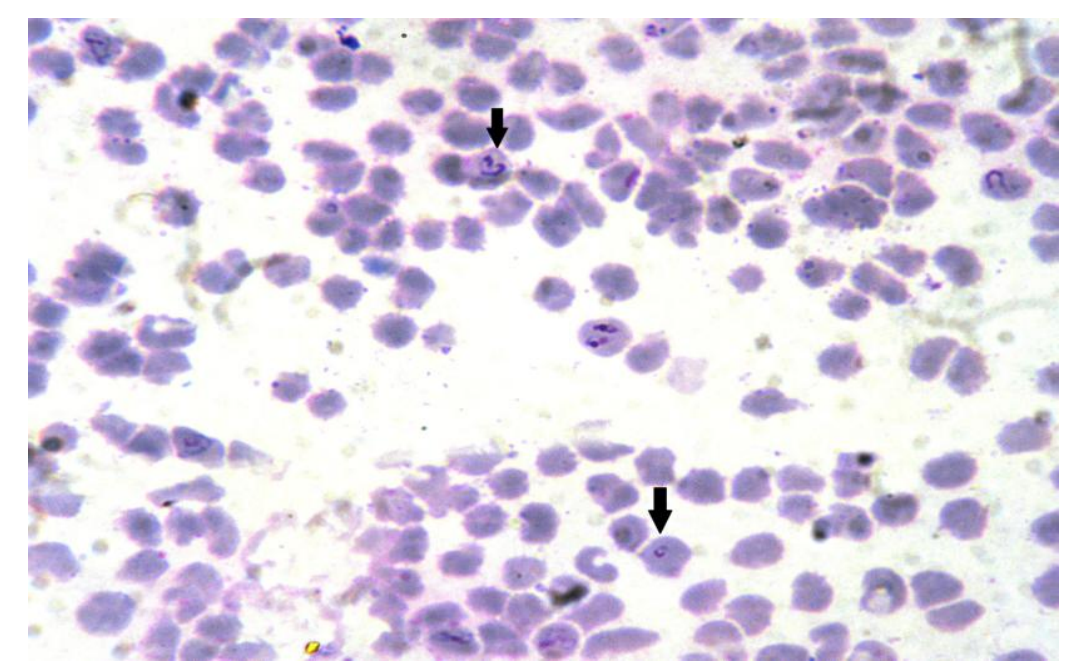

Theileriosis and babesiosis were commonly observed hemoprotozoan infections which caused high degree of morbidity and mortality in cross-bred and exotic animals (Mehlhorn et $a l .$, 1994). These diseases were transmitted by Ixodid ticks. The causative agent of tropical theileriois was Theileria annulata and was transmitted by Hyalomma ticks. Bovine babesiosis was mainly caused by Babesia bigemina, Babesia bovis and Babesia divergens and were transmitted by Boophilus ticks. Theileriosis was characterized by fever, swelling of superficial lymphodes, pale and icterus mucous membranes and Babesiosis was characterized by fever, hemoglobinuria, anemia and jaundice (Radostits et al., 2007). Most common technique for detection of these haemoparasites was microscopic examination of blood smears stained with Giemsa. Examination of Giemsa stained 
peripheral blood smear revealed the presence of piroplasms of Babesia bigemina as characteristic pear shaped bodies joined at an acute angle, besides amoeboid shaped piroplasms along with ring shaped piroplasms of Theileria annulata (Singh, 2011). This technique was usually adequate for detection of acute infections (Friedhoff and Bose, loc.cit). Chemotherapy of theileriosis and babesiosis include buparvaquone at $2.5 \mathrm{mg} / \mathrm{kg}$ BW (Radostits et al., loc.cit) and diminazene aceturate at $3-5 \mathrm{mg} / \mathrm{kg} \mathrm{BW}$ (Mosqueda et al., 2012), respectively. Infection with tickborne protozoan parasite, Babesia bigemina caused erythrocytic destruction and resulted in hemoglobinuria. In an untreated cases, continuing erythrocyte destruction might lead to severe anemia and in such cases, there was fall in packed cell volume below 10 to 12 percent. In cases with PCV below 10 percent, blood transfusion is indicated and that would probably save the animal's life (Soldan, 1999). Hence, concurrent infection of babesiosis and theileriosis associated with high degree of morbidity and mortality, timely therapeutic management with antiprotozoal drugs and supportive therapy along with timely blood transfusion leads to uneventful recovery.

\section{References}

Benjamin, M. M., 1998. Urinalysis. In: Outline of Veterinary Clinical Pathology, 3rd Edition, Kalyani Publishers, Ludhiana, pp. 180-212.

Friedhoff, K. and Bose, R. 1994. Recent developments in diagnostics of some tick-borne diseases. In: Uilenberg, G., Permin, A., Hansen, J.W. (Eds.), Use of Applicable Biotechnological Methods for Diagnosing Haemoparasites. Proceedings of the Expert Consultation, Merida, Mexico, 1993. Food and Agriculture Organisation of the United Nations (FAO), Rome, Italy, pp. 46-57.

Mehlhorn, H. and Schein, E. 1984. The piroplasms: life cycle and sexual stages. Adv. Parasitol., 23: 37-103.

Mehlhorn, H., E. Schein and Ahmed, J. S 1994. Theileria. In: Kreier JP (eds) Parasitic Protozoa, part 2, vol 7. Academic Press, San Diego, Calif., pp. 217-304.

Mosqueda, J., A. Olvera-Ramirez, G. AguilarTipacamu and Canto, G. J. 2012. Current advances in detection and treatment of babesiosis. Current Med. Chemis., 19:1504-1518.

Radostits, O.M., C.C. Gay, K.W. Hinchcliff and Constable, P. D. 2007. Veterinary Medicine. A textbook of the diseases of cattle, horses, sheep, pigs and goats. $10^{\text {th }}$ ed., W.B. Saunders Elsevier, Philadelphia. pp .1483-1497 \& 15301531.

Singh. H, N.K. Singh, N. Chand, S. Deshmukh, Jyoti, R. S. Singh and Rath, S. S. 2011. Concurrent infection of Babesia bigemina and Theileria annulata in Holstein Friesian cow: A case report. J. of Vet. Parasitolo., 25: 8283.

Soldan. A. 1999. Blood transfusions in cattle. In Pract., 21: 590-595.

\section{How to cite this article:}

Ramyadevi, R. and Ponnuswamy, K. K. 2020. Successful Therapeutic Management of Concurrent Infection of Babesiosis and Theileriosis in a Holstein Friesian Calf. Int.J.Curr.Microbiol.App.Sci. 9(08): 1226-1228. doi: https://doi.org/10.20546/ijcmas.2020.908.137 